\title{
CORRESPONDENCE
}

\section{Heterogeneity and other problems in a pooled analysis of}

\section{snus use and mortality [version 1; peer review: 2 approved]}

\author{
Brad Rodu (D1,2, Nantaporn Plurphanswat ${ }^{1}$ \\ 1James Graham Brown Cancer Center, University of Louisville, Louisville, KY, 40202, USA \\ ${ }^{2}$ Department of Medicine, School of Medicine, University of Louisville, Louisville, KY, 40202, USA
}

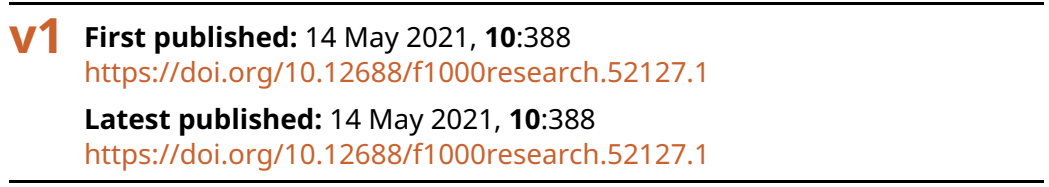

\section{Abstract}

A recent analysis of Swedish snus use and mortality combined eight Swedish datasets and found that exclusive Swedish male snus users have statistically significant increased mortality from all causes, cardiovascular diseases and other causes. These findings, from the Swedish Collaboration on Health Effects of Snus Use, are in sharp contrast with previous pooled results from the same group. The discrepant results may be indicative of unresolved statistical problems that haven't been addressed by the collaboration authors in any of their studies.

The most important problem is unresolved heterogeneity among the eight cohorts, which we describe in detail, and we show how the use of the random effects method by the authors was not sufficient. We explain why the tables in the article are uninformative, and we demonstrate why the exclusion of smokers in the analysis was not validated and eliminated important information. Finally, we strongly recommend some straightforward and easily implemented corrective measures.

\section{Keywords}

snus use, smokeless tobacco, mortality follow-up study, Swedish

Collaboration on Health Effects of Snus Use, heterogeneity

\section{Open Peer Review \\ Approval Status \\ 1 2 \\ version 1 \\ 14 May 2021
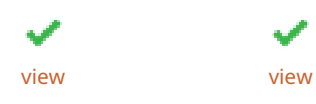

1. Marewa Glover (D), Centre of Research

Excellence: Indigenous Sovereignty \&

Smoking (COREISS), Auckland, New Zealand

2. Tord Finne Vedoy , Norwegian Institute of Public Health, Oslo, Norway

Any reports and responses or comments on the article can be found at the end of the article. 
Corresponding author: Brad Rodu (brad.rodu@louisville.edu)

Author roles: Rodu B: Conceptualization, Writing - Original Draft Preparation, Writing - Review \& Editing; Plurphanswat N: Conceptualization, Writing - Original Draft Preparation, Writing - Review \& Editing

Competing interests: The authors are supported by unrestricted grants from tobacco manufacturers to the University of Louisville, and by the Kentucky Research Challenge Trust Fund. The sponsors had no knowledge of this work, and therefore had no input or other influence in the design, analysis, interpretation of results, or in the preparation of and decision to submit the correspondence. The authors have no financial or other personal relationship with regard to the sponsors.

Grant information: This work is supported by the Kentucky Research Challenge Trust Fund.

The funders had no role in study design, data collection and analysis, decision to publish, or preparation of the manuscript.

Copyright: ( 2021 Rodu B and Plurphanswat N. This is an open access article distributed under the terms of the Creative Commons Attribution License, which permits unrestricted use, distribution, and reproduction in any medium, provided the original work is properly cited.

How to cite this article: Rodu B and Plurphanswat N. Heterogeneity and other problems in a pooled analysis of snus use and

mortality [version 1; peer review: 2 approved] F1000Research 2021, 10:388 https://doi.org/10.12688/f1000research.52127.1

First published: 14 May 2021, 10:388 https://doi.org/10.12688/f1000research.52127.1 


\section{Introduction}

A recent analysis by Byhamre et al. of Swedish snus use and mortality combined eight Swedish datasets and found that exclusive Swedish male snus users have statistically significant increased mortality from all causes (adjusted hazard ratio, $\mathrm{aHR}=1.28,95 \%$ confidence interval, $\mathrm{CI}=1.20-1.35)$, cardiovascular diseases $(\mathrm{aHR}=1.27, \mathrm{CI}=1.15-1.41)$ and other causes $(\mathrm{aHR}=1.37, \mathrm{CI}=1.24-1.52)$, as well as a $12 \%$ elevation in cancer mortality $(\mathrm{aHR}=1.12, \mathrm{CI}=1.00-1.26){ }^{1}$ These findings, from the Swedish Collaboration on Health Effects of Snus Use, are in sharp contrast with previous pooled results from the same group that found no significant increases in incidence and/or mortality from acute myocardial infarction, ${ }^{2}$ stroke, ${ }^{3}$ atrial fibrillation, ${ }^{4}$ pancreatic cancer, ${ }^{5}$ oral cancer ${ }^{6}$ and colorectal cancer. ${ }^{7}$ The latter study did report a significant association of exclusive current snus use and rectal cancer $(\mathrm{aHR}=1.38, \mathrm{CI}=1.07-1.77)^{7}$

We are concerned that the discrepant results between the latest analysis and previous published studies may be indicative of unresolved statistical problems that haven't been addressed by the collaboration authors in any of their studies. These problems include unresolved heterogeneity among the eight cohorts, for which the random effects method the authors used was not a sufficient remedy. In addition, the tables in the article are uninformative, and the exclusion of smokers in the analysis was not validated and eliminated important information. There are straightforward and easily implemented corrective measures that the authors could undertake.

\section{The pooled cohorts are highly heterogeneous}

First, the cohorts in the study are heterogeneous, as our Table 1 demonstrates. The eight cohorts were all described as "population-based," but only four fit that description, and two of those were duplicative. The population-based cohorts were from: (a) the most populous Swedish County - the Stockholm Public Health Cohort (StPHC); (b) a Diet and Cancer Study in the third most populous city, Malmö; (MDCS) (c) the Scania Public Health Cohort (ScPHC), which contains Malmö; and (d) the Multinational Monitoring of Trends and Determinants in Cardiovascular Disease (MONICA) cohort, from rural Västerbotten and Norbotten Counties, which make up 34\% of Sweden's land area but only $5 \%$ of its population. The other four cohorts were convenience samples (the first three are from throughout Sweden). They consisted of: (a) the construction worker cohort (CWC); (b) participants in a charity walk, the National March Cohort (NMC); (c) twins born from 1926 to 1958 (Screening Across the Lifespan Twin, or SALT, Study); and (d) employees in three counties (Work, Lipids and Fibrinogen, WOLF study), which included Stockholm.

The heterogeneity of the samples is further illustrated by other characteristics described in Table 1, including the mean age at death. It was 61 years among employees, which was much lower compared not only with the other cohorts, but with men in Sweden during the same recruitment and follow-up periods. ${ }^{8}$ Other diverse variables were the prevalence of smoking and snus use. As we discuss in detail later, Byhamre et al. ${ }^{1}$ excluded smokers from their analysis, but didn't justify their choice, which is not innocuous and contributes to the estimates that the authors conclusions are based upon. They also, misleadingly, included these omitted observations in their table describing their cohorts. So, we point out that smoking and snus use prevalence varied from very low (7\% and 9\%, respectively) among charity walkers to very high (46\% and $27 \%$ ) among construction workers.

The construction workers account for $72-73 \%$ of both the sample and the number of deaths. Their mean enrollment age was 34 years, which was vastly different than four other cohorts that recruited men aged $50+$ years. The first mortality follow-up study of snus use involving this cohort, published in $1994,{ }^{9}$ had an unusual and unresolved distribution of excess deaths. ${ }^{10}$

Pooled analyses are intended to yield results that are generalizable to the pool. However, the vast differences in the characteristics of the cohorts that have been disclosed raise a concern whether the findings are interpretable as population estimates. As discussed, only four cohorts were from population-based samples, and three enrolled very small numbers. The participant selection process in the vastly larger convenience sample cohorts is the dominant influence of this study's results and, given that the employee cohort is by far the largest and makes up a large majority of the pooled sample, the very different snus use and mortality in this cohort is reason alone to question the external validity of the results even if they are correct, which we do not think is the case.

\section{The methodology was flawed}

Byhamre et al. ${ }^{1}$ used a random-effects method to account for the variation in mortality across cohorts, in which they assumed that there is no correlation between individual cohorts and snus use. However, that method also assumes that unmeasured factors specific to a cohort are unrelated to either snus use or mortality, which is almost surely unlikely to hold in practice. For example, as we show in Table 1, the mean age at enrollment in the four population-based cohorts was 48 to 59 years, compared with 34 years in the construction workers, the latter accounting for over $70 \%$ of the combined 


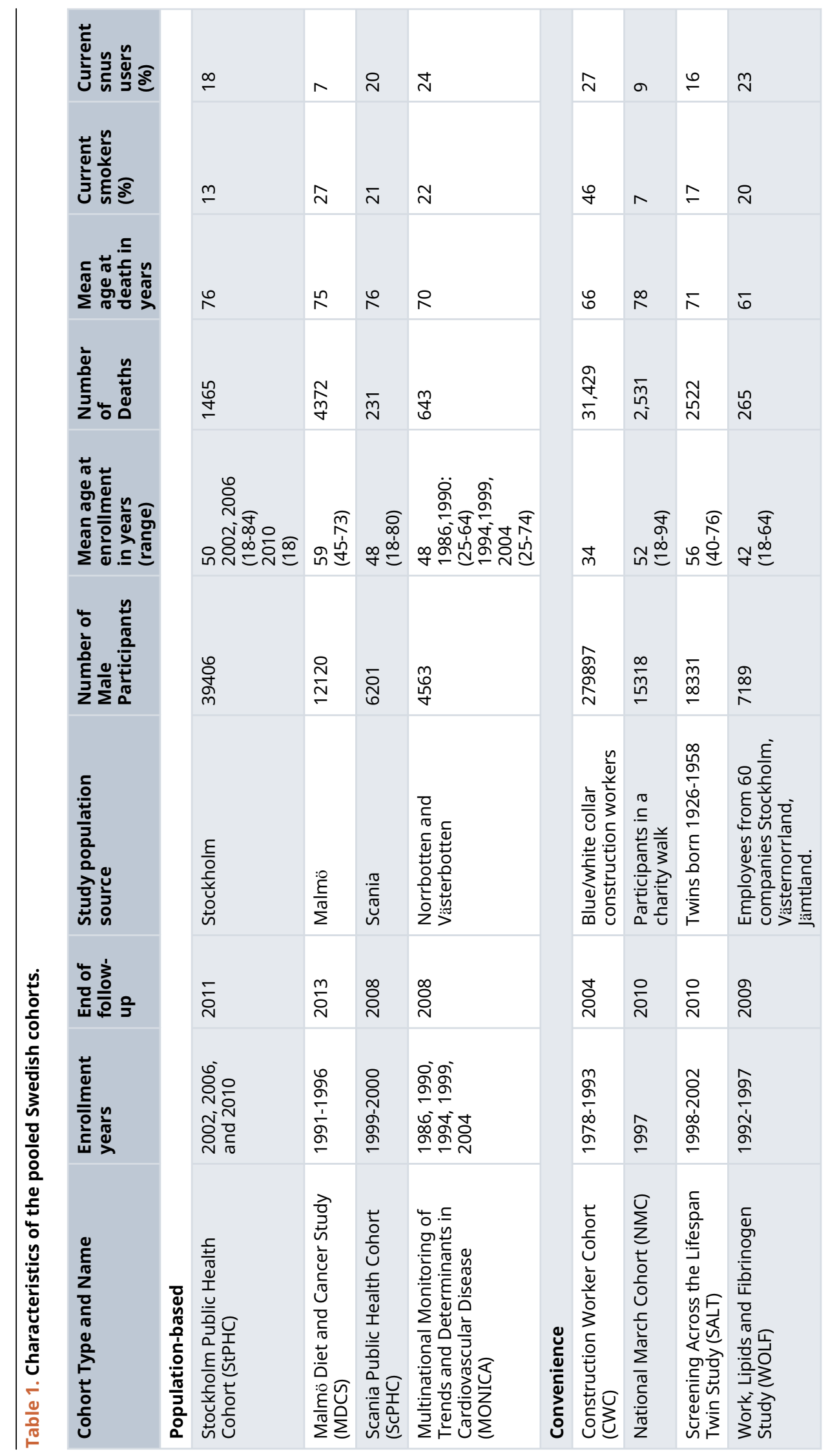


sample. Furthermore, the mean age at death was highly variable, ranging from 61 years in the employee sample and 66 years in construction workers to $70+$ years in the other cohorts.

At a minimum, the authors could have addressed these problems by other methods ${ }^{11,12}$ providing the following information:

- Estimate adjusted hazard ratios for each cohort separately. This provides information about which cohorts are driving the pooled results, and it is especially important when over $70 \%$ of the sample was in the construction worker cohort.

This also addresses the selection problem tied to cohort populations with considerably different age distributions and demographic characteristics. Consider the four cohorts (two population-based and two convenience samples) that enrolled participants at or above a mean age of 50 years. By definition, everyone in these cohorts had already lived at least 50 years and was alive at enrollment. Now compare them with the construction worker cohort, which was enrolled at a mean age of 34 years. Sixteen years later, at age 50, less than $100 \%$ of this cohort was still living and contributing person-years, which makes the survivors fundamentally different people compared with those who were enrolled after age 50. The pooled analysis methodology from Byhamre et al. ignored this serious problem, and their outcomes may have been significantly biased.

- Estimate a "fixed effects" model by assigning indicator (dummy) variables for cohorts and interacting the cohort variables with age and education. This will allow correlation between the cohorts and snus use and between the cohorts and mortality. Furthermore, the interaction between cohorts and age would allow the effect of age on mortality to differ by cohorts.

\section{The tables are uninformative}

Byhamre et al. provided the "Characteristics of included cohorts..." in Table 1 of their publication, and we have used some of this information to illustrate heterogeneity and other problems. ${ }^{1}$ However, this table does not reflect Byhamre et al.'s actual analytic sample $(n=169,103)$, because it includes the characteristics of all male participants from the eight cohorts $(n=383,025)$, including ever regular smokers and others that the authors excluded. Simply put, including information on 214,000 participants that aren't in the analysis is not only uninformative, it is misleading and must be revised.

Although Byhamre et al.'s Table 2 provides information on some baseline characteristics in the analytic sample, it presents the pooled characteristics, which is uninformative because it further conceals large differences between the cohorts. For example, variations in enrollment ages across the cohorts have substantial impact on BMI, physical activity, and alcohol use that may overwhelm and obfuscate any differences due to snus use. The authors should have reported the baseline characteristics according to cohorts, and cohorts by snus use.

Furthermore, the authors did not provide adequate definitions for snus use, the sole exposure of interest in the study. Thus, questionnaires containing actual questions about snus use from each cohort is a prerequisite. According to the authors, baseline snus use was not consistent throughout the cohorts. Some studies did not have information on former snus use, while others did not have information on amount and/or duration.

\section{The exclusion of smokers was not validated and eliminates important information}

The authors excluded participants "reporting ever regular use of cigarettes" (emphasis added), which was intended "to eliminate potential residual effects", a vague reference to an unspecified statistical problem. The authors provided no foundation for this choice and no evidence that their objective was met. Furthermore, snus users who were less-thanregular cigarette smokers - those with a smoking history and some dual users - were still in the pooled analytic cohort. ${ }^{13}$ Less-than-regular smoking is likely because of the lower social acceptability of smoking vis-à-vis snus in Sweden. ${ }^{14,15}$

The authors could validate whether their analytic tactic excluded smoking by reporting the results for two disease categories: cancer of the trachea, bronchus, and lung (ICD-10 codes C33-C34) and chronic lower respiratory diseases (J40-J47). If they truly eliminated smokers from their analyses, they should not have found any significantly increased hazard ratios for these diseases among snus users.

Excluding "ever regular" smokers is a routine practice in Swedish follow-up studies of snus use, but it also eliminates a lot of valuable information and may bias the results. First, partial exclusion of an exposure closely associated with snus use 
precludes assessment of interactions. Second, the findings on snus use in this and similar studies completely lack context with respect to the effects of smoking on mortality. ${ }^{16}$

\section{Summary}

In conclusion, Byhamre et al. ${ }^{1}$ published an analysis with several flaws that render their results uninformative and possibly misleading. The authors should publish revised tables and re-estimate their analyses as described above to address these problems. These measures are readily achievable, and the validity of the results will be considerably enhanced.

\section{Data availability}

No data is associated with this article.

1. Byhamre ML, Araghi M, Alfredsson L, et al.: Swedish snus use is associated with mortality: a pooled analysis of eight prospective studies. Int J Epidemiol. 2021; 49: 2041-2050. PubMed Abstract | Publisher Full Text | Free Full Text

2. Hansson J, Galanti MR, Hergens M-P, et al.: Use of snus and acute myocardial infarction: pooled analysis of eight prospective studies. Eur J Epidemiol. 2012; 27: 771-779. PubMed Abstract | Publisher Full Text

3. Hansson J, Galanti MR, Hergens M-P, et al.: Snus (Swedish smokeless tobacco) use and risk of stroke: pooled analyses of incidence and survival. J Int Med. 2014; 276: 87-95. PubMed Abstract | Publisher Full Text

4. Hergens M-P, Galanti R, Hansson J, et al.: Use of Scandinavian moist smokeless tobacco (snus) and the risk of atrial fibrillation. Epidemiol. 2014; 25: 872-876.

PubMed Abstract | Publisher Full Text

5. Araghi M, Galanti MR, Lundberg M: Use of moist snuff (snus) and pancreatic cancer: pooled analysis of nine prospective observational studies. Int J Cancer. 2017; 141: 687-693. PubMed Abstract | Publisher Full Text

6. Araghi M, Galanti MR, Lundberg M: No association between moist oral snuff (snus) use and oral cancer: pooled analysis of nine prospective observational studies. Scand J Public Health. 2020, May 28: 1403494820919572 . Online ahead of print. PubMed Abstract | Publisher Full Text

7. Araghi M, Galanti MR, Lundberg M: Smokeless tobacco (snus) use and colorectal cancer incidence and survival: results from nine pooled cohorts. Scand J Public Health. 2017; 45: 741-748. PubMed Abstract | Publisher Full Text

8. Statistics Sweden: Life expectancy. 1751-2019. Reference Source
9. Bolinder G, Alfredsson L, Englund A, et al.: Smokeless tobacco use and increased cardiovascular mortality among Swedish construction workers. Am J Public Health. 1994; 84 399-404.

PubMed Abstract | Publisher Full Text | Free Full Text

10. Rodu B, Cole P: Excess Mortality in Smokeless Tobacco Users Not Meaningful (Letter). Am J Public Health. 1995; 85: 118. PubMed Abstract | Publisher Full Text | Free Full Text

11. Blettner M, Sauerbrei W, Schlehofer B, et al.: Traditional reviews, meta-analyses and pooled analyses in epidemiology. Int J Epidemiol. 1999; 28: 1-9.

PubMed Abstract | Publisher Full Text

12. Friedenreich CM: Methods for Pooled Analyses of Epidemiologic Studies. Epidemiol. 1993; 4: 295-302. PubMed Abstract | Publisher Full Text

13. Rodu B, Stegmayr B, Nasic S, et al.: Impact of smokeless tobacco use on smoking in northern Sweden. J Int Med. 2002; 252: 398-404. PubMed Abstract | Publisher Full Text

14. Norberg M, Malmberg $\mathrm{G}, \mathrm{Ng} \mathrm{N}$, et al.: Who is using snus? - Time trends, socioeconomic and geographic characteristics of snus users in the ageing Swedish population. BMC Public Health. 2011; 11: 929. PubMed Abstract | Publisher Full Text | Free Full Text

15. Ramström L, Borland R, Wikmans T: Patterns of Smoking and Snus Use in Sweden: Implications for Public Health. Int J Environ Res Public Health. 2016; 13(11): 1110. Published 2016 Nov 9. PubMed Abstract | Publisher Full Text | Free Full Text

16. Rodu B, Plurphanswat N: Mortality among male smokers and smokeless tobacco users in the U.S. Harm Red J. 2019; 16. Reference Source Accessed 8 February 2021 


\section{Open Peer Review}

\section{Current Peer Review Status:}

\section{Version 1}

Reviewer Report 11 March 2022

https://doi.org/10.5256/f1000research.55362.r124659

(C) 2022 Vedoy T. This is an open access peer review report distributed under the terms of the Creative Commons Attribution License, which permits unrestricted use, distribution, and reproduction in any medium, provided the original work is properly cited.

\section{Tord Finne Vedoy}

Department of Alcohol Drugs and Tobacco, Norwegian Institute of Public Health, Oslo, Norway

The debate on the degree to which snus is associated with increased morbidity or mortality, and how, has been going on for some time.

Although the discussion may seem most relevant for those relative few who use snus or live in a society where snus use is common, the debate should interest tobacco researchers generally for (at least) two reasons: First, snus is one of the products that has been both a popular and longlasting harm reducing alternative to cigarette smoking. Correct estimates of the harms associated with snus use are necessary to assess the harm-reducing potential of snus use. Second, estimating the harmfulness of snus use requires that researchers take into account the use of two distinct but related tobacco products. Both these dimensions can inform similar discussions on the harmreducing potentials of other and more recent nicotine products, for example, e-cigarettes.

Because few countries have a long history of snus use, there is limited data on individual snus and other tobacco use patterns and health outcomes from national registers. Consequently, many of the studies concerning the association between snus use and registered mortality has been based on the same set of (Swedish) cohorts and examined by the same researchers.

I find that the issues raised by Rodu and Plurphanswatare are warranted and should be relatively easy to address. A revised study by Byhamre et al. would be of great interest to both debates on snus use and public health and snus use and harm reduction.

Is the rationale for commenting on the previous publication clearly described? Yes

Are any opinions stated well-argued, clear and cogent? Yes

Are arguments sufficiently supported by evidence from the published literature or by new data and results?

Yes 


\section{Is the conclusion balanced and justified on the basis of the presented arguments? Yes}

Competing Interests: No competing interests were disclosed.

Reviewer Expertise: Social explanations of tobacco use and social inequality in tobacco use.

\section{I confirm that I have read this submission and believe that I have an appropriate level of expertise to confirm that it is of an acceptable scientific standard.}

Reviewer Report 03 June 2021

https://doi.org/10.5256/f1000research.55362.r85432

(C) 2021 Glover M. This is an open access peer review report distributed under the terms of the Creative Commons Attribution License, which permits unrestricted use, distribution, and reproduction in any medium, provided the original work is properly cited.

\section{Marewa Glover}

Centre of Research Excellence: Indigenous Sovereignty \& Smoking (COREISS), Auckland, New Zealand

The letter 'Heterogeneity and other problems in a pooled analysis of snus use and mortality' raises concerns about the method used in a paper by Byhamre et al., 2021 that was published in the International Journal of Epidemiology (Vol.49). The Byhamre et al. paper entitled 'Swedish snus use is associated with mortality: a pooled analysis of eight prospective studies' claimed to have found a positive association between snus use and mortality, where previous studies by the same group had not found this association. Rodu and Plurphanswat outline a range of methodological flaws in the Byhamre et al. analysis.

The critique is straightforward and clearly written. The authors have narrowly focused on the poor use of statistical analysis methods. They could have gone further.

A more in-depth critical and systematic review of the literature being produced by the Swedish Collaboration on Health Effects of Snus is warranted. Particularly, future reviews should include an analysis of Risk of Bias. It is a minor example, but it is indicative that the authors on the Byhamre et al. paper do not consider the potential bias inherent in their focus on mostly their own previous work. Nine of the Byhamre et al. authors were investigators and authors on one or more of the eight studies the 'pooled' data were extracted from. This is a form of authorship bias.

There is a much larger pool of research that has sought to determine and quantify the risks of snus than indicated by Byhamre et al.'s selection. Future critical reviews would need to identify if selection bias is a further limitation of the Byhamre et al. analysis.

Critiques of published science make an important contribution to science. To ensure knowledge is sound, these types of critiques could even be said to be essential. Seriously flawed studies can mislead policy makers and politicians and lead to decisions about healthcare that could cause 
harm.

Is the rationale for commenting on the previous publication clearly described?

Yes

Are any opinions stated well-argued, clear and cogent?

Yes

Are arguments sufficiently supported by evidence from the published literature or by new data and results?

Yes

Is the conclusion balanced and justified on the basis of the presented arguments?

Yes

Competing Interests: Professor Marewa Glover is Director of the Centre of Research Excellence: Indigenous Sovereignty \& Smoking which was established with a grant from the Foundation for a Smoke-Free World, a US nonprofit 501(c)(3) private foundation with a mission to end smoking in this generation. The Foundation accepts charitable gifts from PMI Global Services Inc. (PMI); under the Foundation's Bylaws and Pledge Agreement with PMI, the Foundation is independent from PMI and the tobacco industry. The contents, selection, and presentation of facts, as well as any opinions expressed herein are the sole responsibility of the author and under no circumstances shall be regarded as reflecting the positions of the Foundation for a Smoke-Free World, Inc. Of relevance to the topic of snus, Professor Glover was a co-author, with Dr CV Phillips, of a systematic review that investigated the 'Potential effects of using noncombustible tobacco and nicotine products during pregnancy' (published in Harm Reduction Journal 2020 17(6):1-12). For over a decade she has advocated for a harm reduction approach to reducing the morbidity and mortality associated with tobacco use.

Reviewer Expertise: Behavioural science; tobacco dependency and cessation; tobacco control; harm reduction; mixed methods from trial design to in-depth qualitative; narrative and systematic reviews.

I confirm that I have read this submission and believe that I have an appropriate level of expertise to confirm that it is of an acceptable scientific standard. 
The benefits of publishing with F1000Research:

- Your article is published within days, with no editorial bias

- You can publish traditional articles, null/negative results, case reports, data notes and more

- The peer review process is transparent and collaborative

- Your article is indexed in PubMed after passing peer review

- Dedicated customer support at every stage

For pre-submission enquiries, contact research@f1000.com 